\title{
A note on the solutions of the Ginsparg-Wilson relation
}

\author{
Ting-Wai Chiu ${ }^{1}$ \\ Department of Physics, National Taiwan University, Taipei, Taiwan 106, People's Republic of China
}

Received 3 November 1999; received in revised form 18 November 1999; accepted 22 December 1999

Editor: M. Cvetič

\begin{abstract}
The role of $R$ in the solutions of the Ginsparg-Wilson relation is discussed. (c) 2000 Published by Elsevier Science B.V. All rights reserved.
\end{abstract}

PACS: 11.15.Ha; 11.30.Rd; 11.30.Fs

In this note, we would like to clarify some seemingly subtle issues pertaining to the role of $R$ in the solutions of the Ginsparg-Wilson relation [1]

$D \gamma_{5}+\gamma_{5} D=2 D \gamma_{5} R D$.

Here $R$ is a positive definite hermitian operator which is local in the position space and trivial in the Dirac space. Since we can sandwich (1) by a left multiplier $\sqrt{R}$ and a right multiplier $\sqrt{R}$ on both sides of (1), and define $D^{\prime}=\sqrt{R} D \sqrt{R}$, then $D^{\prime}$ satisfies

$D^{\prime} \gamma_{5}+\gamma_{5} D^{\prime}=2 D^{\prime} \gamma_{5} D^{\prime}$

which is in the same form of Eq. (1) with $R=\mathbb{1}$. This seems to suggest that one can set $R=\mathbb{1}$ in the GW relation (1) and completely ignore the $R$ dependence in the general solution of the GW relation. However, as we will see, if $R$ is set to be the identity operator from the beginning, then some of the salient

\footnotetext{
${ }^{1}$ E-mail: twchiu@phys.ntu.edu.tw
}

features of the general solution may be easily overlooked.

First, let us review some basics of the GW relation. The general solution to the GW relation (1) can be written as $[2,3]$

$$
\begin{aligned}
D & =D_{c}\left(\mathbb{1}+R D_{c}\right)^{-1} \\
& =\left(\mathbb{1}+D_{c} R\right)^{-1} D_{c},
\end{aligned}
$$

where $D_{c}$ is any chirally symmetric Dirac operator, i.e.,

$D_{c} \gamma_{5}+\gamma_{5} D_{c}=0$.

In order to have $D$ reproduce the continuum physics, $D_{c}$ is required to satisfy the necessary physical constraints [3]. The general solution of $D_{c}$ has been investigated in Ref. [4]. Conversely, for any $D$ satisfying the GW relation (1), there exists the chirally symmetric $D_{c}$

$$
\begin{aligned}
D_{c} & =D(\mathbb{I}-R D)^{-1} \\
& =(\mathbb{1}-D R)^{-1} D .
\end{aligned}
$$


We usually require that $D$ also satisfies the hermiticity condition ${ }^{2}$

$D^{\dagger}=\gamma_{5} D \gamma_{5}$.

Then $D_{c}$ also satisfies the hermiticity condition $D_{c}^{\dagger}$ $=\gamma_{5} D_{c} \gamma_{5}$, since $R$ is hermitian and commutes with $\gamma_{5}$. The hermiticity condition together with the chiral symmetry of $D_{c}$ implies that $D_{c}$ is anti-hermitian. Thus there exists one to one correspondence between $D_{c}$ and a unitary opertor $V$ such that

$D_{c}=(\mathbb{1}+V)(\mathbb{1}-V)^{-1}$,

$V=\left(D_{c}-\mathbb{1}\right)\left(D_{c}+\mathbb{1}\right)^{-1}$.

where $V$ also satisfies the hermiticity condition $V^{\dagger}$ $=\gamma_{5} V \gamma_{5}$. Then the general solution (3) can be written as $[2,3]$

$$
\begin{aligned}
D & =(\mathbb{1}+V)[(\mathbb{1}-V)+R(\mathbb{1}+V)]^{-1} \\
& =[(\mathbb{1}-V)+(\mathbb{1}+V) R]^{-1}(\mathbb{1}+V) .
\end{aligned}
$$

On the other hand, if one starts from Eq. (2), then its solution is

$D^{\prime}=\frac{1}{2}(\mathbb{I}+V)$

which agrees with (8) with $R=\mathbb{1}$. Then using the relation $D^{\prime}=\sqrt{R} D \sqrt{R}$, one obtains

$D=\frac{1}{\sqrt{2 R}}(\mathbb{1}+V) \frac{1}{\sqrt{2 R}}$.

However, (11) is in contradiction with (8) since the $R$ dependence in (11) can be factored out completely while that of (8) can not. So, one of them can not be true in general. If we take the limit $R \rightarrow 0$, then (8) gives that $D \rightarrow(\mathbb{1}+V)(\mathbb{1}-V)^{-1}=D_{c}$, but (11) implies that $D \rightarrow \infty$. Since $D_{c}$ is well defined (without poles) in the trivial gauge sector, it follows that (11) can not be true in general. The fallacy in (11) is due to the assumption that $D^{\prime}$ is independent of $R$. From (8), one can derive the following formula

$D=\frac{1}{\sqrt{2 R}}\left(\mathbb{1}+V^{\prime}\right) \frac{1}{\sqrt{2 R}}$,

\footnotetext{
${ }^{2}$ This implies that $\operatorname{det}(D)$ is real and non-negative.
}

where

$$
\begin{aligned}
V^{\prime}= & {\left[(\mathbb{1}+V) \sqrt{R}+(\mathbb{1}-V) \frac{1}{\sqrt{R}}\right]^{-1} } \\
& \times\left[(\mathbb{1}+V) \sqrt{R}-(\mathbb{1}-V) \frac{1}{\sqrt{R}}\right] \\
= & {\left[\sqrt{R}(\mathbb{1}+V)-\frac{1}{\sqrt{R}}(\mathbb{1}-V)\right] } \\
& \times\left[\sqrt{R}(\mathbb{1}+V)+\frac{1}{\sqrt{R}}(\mathbb{1}-V)\right]^{-1}
\end{aligned}
$$

which is unitary and depends on $R$. This shows that $D^{\prime}$ actually depends on $R$ and equals to $\frac{1}{2}\left(\mathbb{1}+V^{\prime}\right)$ rather than (10). Therefore it is erroneous to write the general solution of the GW relation in the form of (11), in which $V^{\prime}$ is replaced by $V$. Consequently, (11) may mislead one to infer that $R$ does not play any significant roles in the locality of $D$, in particular when $R$ is proportional to the identity operator. However, $R$ indeed plays a very important role in determining the locality of $D$. Let us consider $R=$ $r \mathbb{1}$ with $r>0$. When $r \rightarrow 0, D \rightarrow D_{c}$ which must be nonlocal if $D_{c}$ is free of species doubling and has the correct behavior in the classical continuum limit [5]. As the value of $r$ moves away from zero and goes towards a finite value, $D$ may change from a non-local operator to a local operator. This has been demonstrated in Refs. [6,7]. Therefore, the general solution (3) of the GW relation can be regarded as a topologically invariant transformation (i.e., index $(D)$ $=\operatorname{index}\left(D_{c}\right)$ ) which can transform a nonlocal $D_{c}$ into a local $D$. Conversely, the transformation (5) can transform a local $D$ into the non-local $D_{c}$. For a given $D_{c}$, the set of transformations, $\{\mathscr{T}(R): D=$ $\left.D_{c}\left(\mathbb{1}+R D_{c}\right)^{-1}\right\}$, form an abelian group with parameter space $\{R\}$ [7]. In general, for any lattice Dirac operator $D$ (not necessarily satisfying the GW relation), we can use the topologically invariant transformation $D^{\prime}=D(\mathbb{1}+R D)^{-1}$ to manipulate its locality.

The next question is whether we can gain anything (e.g., improving the locality of $D$ ) by using another functional form of $R$ rather than the simplest choice $R=r \mathbb{1}$. We investigate this question by nu- 
merical experiments. For simplicity, we consider the Neuberger-Dirac operator [8]

$D_{h}=\mathbb{1}+V$,

$V=D_{w}\left(D_{w}^{\dagger} D_{w}\right)^{-1 / 2}$,

where $D_{w}$ is the Wilson-Dirac fermion operator with negative mass -1

$D_{w}=-1+\frac{1}{2}\left[\gamma_{\mu}\left(\nabla_{\mu}^{*}+\nabla_{\mu}\right)-\nabla_{\mu}^{*} \nabla_{\mu}\right]$,

where $\nabla_{\mu}$ and $\nabla_{\mu}{ }^{*}$ are the forward and backward difference operators defined in the following,

$$
\begin{aligned}
& \nabla_{\mu} \psi(x)=U_{\mu}(x) \psi(x+\hat{\mu})-\psi(x), \\
& \nabla_{\mu}^{*} \psi(x)=\psi(x)-U_{\mu}^{\dagger}(x-\hat{\mu}) \psi(x-\hat{\mu}) .
\end{aligned}
$$

The Neuberger-Dirac operator $D_{h}$ satisfies the GW relation (1) with $R=1 / 2$. Then Eq. (5) gives

$D_{c}=2 \frac{\mathbb{1}+V}{\mathbb{1}-V}$.

Substituting (17) into the general solution (3), we obtain

$D=2(\mathbb{1}+V)[(\mathbb{1}-V)+2 R(\mathbb{1}+V)]^{-1}$.

For a fixed gauge background, we investigate the locality of $D(x, y)$ versus the functional form of $R(x, y)$. For simplicity, we consider $D$ in a two dimensional $U(1)$ background gauge field with nonzero topological charge, and we use the same notations for the background gauge field as Eqs. (7)-(11) in Ref. [9]. Although it is impossible for us to go through all different functional forms of $R(x, y)$, we can use the exponential function

$$
R(x, y)=r \exp (-m|x-y|)
$$

as a prototype to approximate other forms by varying the parameters $r$ and $m$. In the limit $m \rightarrow 0, R(x, y)$ is nonlocal, while in the limit $m \rightarrow \infty, R \rightarrow r \mathbb{1}$ which is the most ultralocal. Hence, by varying the value of $m$ from 0 to $\alpha \gg 1$, we can cover a wide range of $R(x, y)$ of very different behaviors.

One of the physical quantities which are sensitive to the locality of $D$ is the anomaly function

$\mathscr{A}_{n}(x)=\operatorname{tr}\left[\gamma_{5}(R D)(x, x)+\gamma_{5}(D R)(x, x)\right]$

which can serve as an indicator of the localness of $D$. Since the Neuberger-Dirac operator is topologi- cally proper for smooth gauge backgrounds, the index of $D$ in (18) is equal to the background topological charge $Q$,

index $(D)=n_{-}-n_{+}=Q$.

This implies that the sum of the anomaly function over all sites on a finite lattice must be equal to two times of the topological charge [7]

$$
\begin{aligned}
\sum_{x} \mathscr{A}_{n}(x) & =2\left(n_{-}-n_{+}\right) \\
& =2 Q \\
& = \begin{cases}\frac{1}{16 \pi^{2}} \sum_{x} \epsilon_{\mu \nu \lambda \sigma} F_{\mu \nu}(x) F_{\lambda \sigma}(x), & d=4 ; \\
\frac{1}{2 \pi} \sum_{x} \epsilon_{\mu \nu} F_{\mu \nu}(x), & d=2 .\end{cases}
\end{aligned}
$$

This is true for any $R$ since the index of $D$ is invariant under the transformation (3), i.e., index $(D)$ $=$ index $\left(D_{c}\right)$. First we consider the gauge configuration with constant field tensors. If $D$ is local, then we can deduce that $\mathscr{A}_{n}(x)$ is constant for all $x$. From (22), it follows that

$$
\begin{aligned}
\mathscr{A}_{n}(x) & =\rho(x) \\
& \equiv \begin{cases}\frac{1}{16 \pi^{2}} \epsilon_{\mu \nu \lambda \sigma} F_{\mu \nu}(x) F_{\lambda \sigma}(x), & d=4 ; \\
\frac{1}{2 \pi} \epsilon_{\mu \nu} F_{\mu \nu}(x), & d=2,\end{cases}
\end{aligned}
$$

where $\rho(x)$ is the Chern-Pontryagin density in continuum. Note that Eq. (23) also implies that $\mathscr{A}_{n}(x)$ is independent of $R$ if $D$ is local. Next we introduce local fluctuations to the constant background gauge field, with the topological charge fixed. Then we expect that (23) remains valid provided that the locality of $D$ is not destroyed by the roughness of the gauge field. Therefore, in general, by comparing the anomaly function $\mathscr{A}_{n}(x)$ at each site with the Chern-Pontryagin density $\rho(x)$, in a prescribed background gauge field, we can reveal whether $D$ is local or not in this gauge background. This provides another scheme to examine the locality of $D$ rather than checking how well $|D(x, y)|$ can be fitted by an exponentially decay function. We will use both 
methods in our investigations. We define the deviation of the anomaly function as

$\delta_{D} \equiv \frac{1}{N_{s}} \sum_{x} \frac{\left|\mathscr{A}_{n}(x)-\rho(x)\right|}{|\rho(x)|}$,

where the summation runs over all sites, and $N_{s}$ is the total number of sites.

In Table 1, we list the deviation of the chiral anomaly function, $\delta_{D}$, versus $R(x, y)$ with parameters $r$ and $m$ defined in Eq. (19), on a $12 \times 12$ lattice, in a constant background gauge field with topological charge $Q=1$. The last two rows with $m \gg 1$ corresponds to $R=r \mathbb{1}$, and they have the smallest deviations. They are both local, which can be checked explicitly by plotting $|D(x, y)|$ versus $|x-y|$, as shown in Fig. 1. For $r=1 / 2$ (the row on top of the last row), it corresponds to the Neuberger-Dirac operator. In the first row, $R(x, y)$ is nonlocal since $m=1 / 2<1$. It produces a nonlocal $D$, as shown in Fig. 2, so the resulting chiral anomaly is very different from the Chern-Pontryagin density and thus $\delta_{D}$ is very large. Now we increase $m$ to 1 , 2 and 5 successively, then $R$ and $D$ both become more and more local, as shown in Fig. 3, thus $\delta_{D}$ becomes smaller and smaller, as shown in the second, third and fourth rows of Table 1 . These results indicate that a nonlocal $R$ does not produce a local $D$, and a local $R$ does not make $D$ more local than that using $R=r$. For $m=5.0$, if we decrease $r$ to 0.1 , then $D$ becomes very nonlocal, as shown in Fig. 4. Consequently, its $\delta_{D}$ (in the fifth row) is about 10 times larger than that of $r=0.5$ (in the fourth row).

Table 1

The deviation of the chiral anomaly function $\delta_{D}$ [Eq. (24)] versus $R(x, y)$ with parameters $r$ and $m$ defined in Eq. (19), on a $12 \times 12$ lattice, in a constant background gauge field with topological charge $Q=1$.

\begin{tabular}{llll}
\hline$r$ & $m$ & $\delta_{D}$ & $\operatorname{index}(D)$ \\
\hline 0.5 & 0.5 & 1.366 & 1 \\
0.5 & 1.0 & 0.5360 & 1 \\
0.5 & 2.0 & 0.2086 & 1 \\
0.5 & 5.0 & $7.209 \times 10^{-3}$ & 1 \\
0.1 & 5.0 & $6.554 \times 10^{-2}$ & 1 \\
0.5 & $\gg 1$ & $3.848 \times 10^{-4}$ & 1 \\
1.0 & $\gg 1$ & $1.618 \times 10^{-4}$ & 1 \\
\hline
\end{tabular}

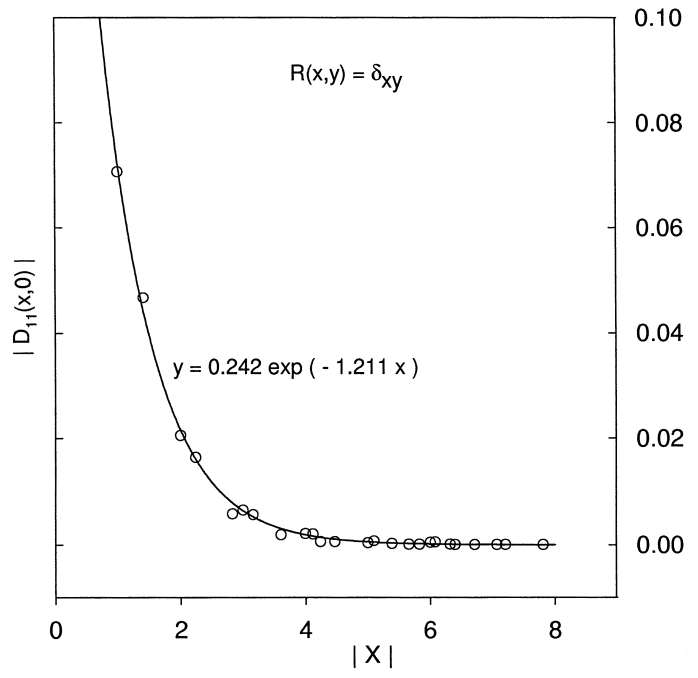

Fig. 1. One of the Dirac components of $D(x, 0),\left|D_{11}(x, 0)\right|$, is plotted as a function of $|x|$ for $R(x, y)=\delta_{x y}$. The lattice is $12 \times 12$ with periodic boundary conditions. The constant background gauge field has topological charge $Q=1$. All data points at the same distance $|x|$ from the origin have been averaged. The solid line is an exponential fit to the data points. The same decay constant also fits very well for all other Dirac components of $D(x, y)$ and for any reference point $y$.

This suggests that on a finite lattice, $r$ cannot be too small, otherwise $D$ will become nonlocal.

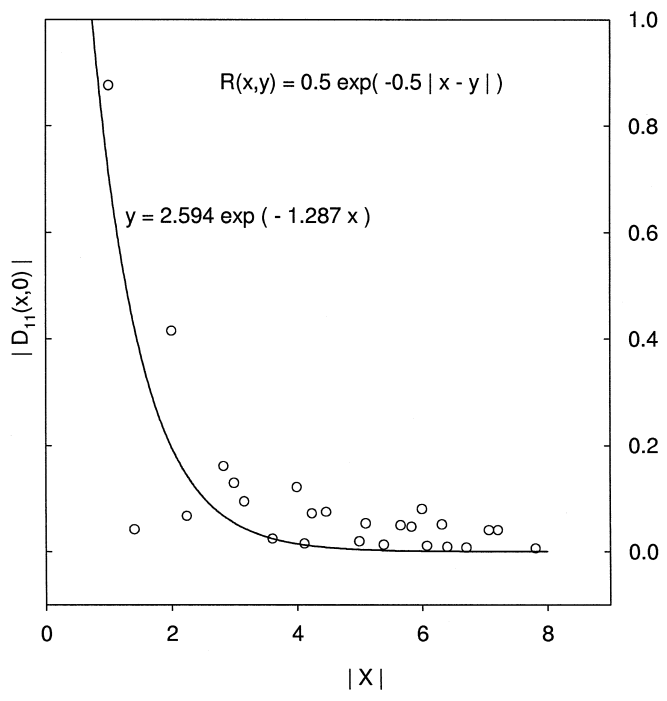

Fig. 2. One of the Dirac components of $D(x, 0)$ is plotted as a function of $|x|$ with $R(x, y)=0.5 \exp (-0.5|x-y|)$. Other descriptions are the same as Fig. 1. The non-localness of $D$ is shown clearly. 


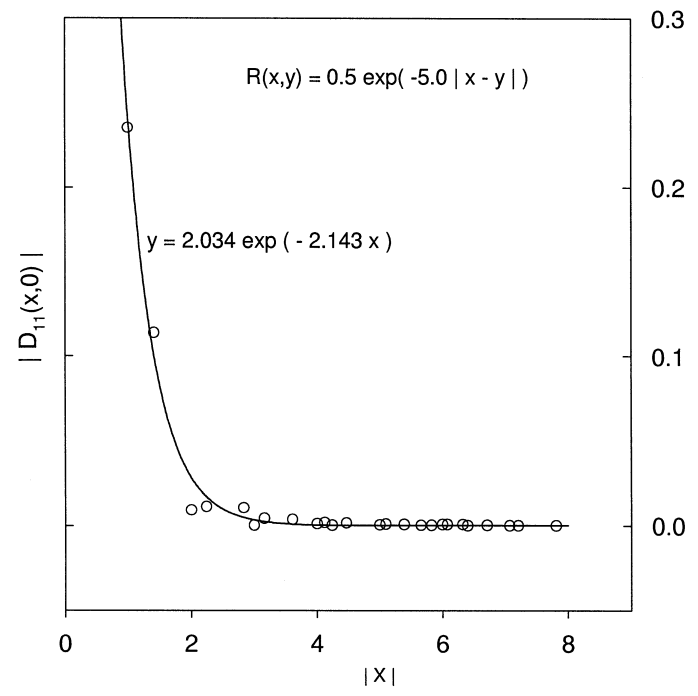

Fig. 3. One of the Dirac components of $D(x, 0)$ is plotted as a function of $|x|$ with $R(x, y)=0.5 \exp (-5.0|x-y|)$. Other descriptions are the same as Fig. 1.

Our numerical results listed in Table 1 as well as those plotted in Figs. 1-4 strongly suggest that we do not gain anything by using other functional forms of $R(x, y)$ than the simplest choice $R(x, y)=r \delta_{x, y}$.

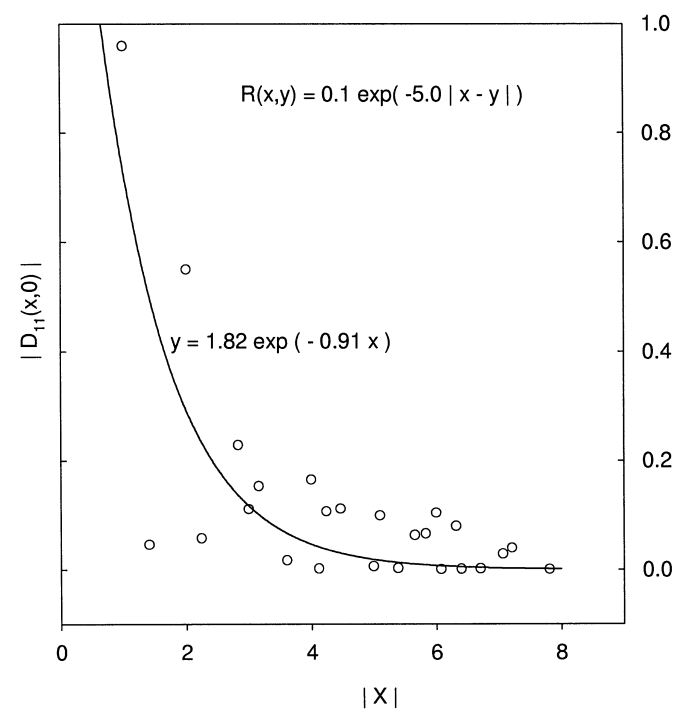

Fig. 4. One of the Dirac components of $D(x, 0)$ is plotted as a function of $|x|$ with $R(x, y)=0.1 \exp (-5.0|x-y|)$. Other descriptions are the same as Fig. 1. The non-localness of $D$ is shown clearly.
However, the value of $r$ plays the important role in determining the localness of $D$. We have also tested other functional forms of $R(x, y)$ as well as many different gauge configurations. The results from all these studies are consistent with the conclusion that the optimal choice for $R$ is $R(x, y)=r \delta_{x, y}$.

Now we come to the question concerning the range of proper values of $r$. We have already known that $r$ cannot be zero or very small, otherwise $D$ is nonlocal. On the other hand, $r$ cannot be too large, otherwise $D$ is highly peaked in the diagonal elements (i.e., $\left.D_{\alpha \beta}(x, y) \sim D_{\alpha \alpha}(x, x) \delta_{\alpha \beta} \delta_{x, y}\right)$, which is unphysical since it does not respond properly to the background gauge field (e.g., the chiral anomaly is incorrect even though the index of $D$ is equal to the background topological charge). In Table 2, we list the deviation of the chiral anomaly function, $\delta_{D}$, versus $R(x, y)=r \delta_{x, y}$, on a $12 \times 12$ lattice (the second column), in a constant background gauge field with topological charge $Q=1$. We see that the proper values of $r$ are approximately in the range $0.5-1.2$, where $D$ can reproduce the continuum chiral anomaly precisely. Next we investigate how the lattice size affects the range of proper values of $r$. The results of $\delta_{D}$ for lattice sizes $16 \times 16$ and $20 \times 20$ are listed in the third and the fourth columns in Table 2. They clearly show that the lower bound of $r$ can be pushed to a smaller value, $\sim 0.2$, when the size of the lattice is increased to $20 \times 20$. Therefore, it suggests that the chiral limit $(r \rightarrow 0$ and

Table 2

The deviation of the chiral anomaly function, $\delta_{D}$ [Eq. (24)], versus $R(x, y)=r \delta_{x, y}$, for lattice sizes, $12 \times 12,16 \times 16$ and $20 \times 20$ respectively. The background gauge field has constant field tensors with topological charge $Q=1$. The index of $D$ is always equal to one in each case.

\begin{tabular}{lllll}
\hline$r$ & $\delta_{D}(12 \times 12)$ & $\delta_{D}(16 \times 16)$ & $\delta_{D}(20 \times 20)$ & $\begin{array}{l}\text { index } \\
(\mathrm{D})\end{array}$ \\
\hline 0.1 & $6.232 \times 10^{-2}$ & $1.792 \times 10^{-2}$ & $4.854 \times 10^{-3}$ & 1 \\
0.2 & $4.818 \times 10^{-3}$ & $6.229 \times 10^{-4}$ & $7.750 \times 10^{-5}$ & 1 \\
0.5 & $3.848 \times 10^{-4}$ & $2.434 \times 10^{-5}$ & $1.559 \times 10^{-6}$ & 1 \\
0.8 & $1.698 \times 10^{-4}$ & $8.268 \times 10^{-6}$ & $4.976 \times 10^{-7}$ & 1 \\
1.0 & $1.618 \times 10^{-4}$ & $6.596 \times 10^{-6}$ & $3.432 \times 10^{-7}$ & 1 \\
1.2 & $3.448 \times 10^{-4}$ & $1.318 \times 10^{-5}$ & $5.308 \times 10^{-7}$ & 1 \\
1.5 & $1.688 \times 10^{-3}$ & $1.243 \times 10^{-4}$ & $8.783 \times 10^{-6}$ & 1 \\
2.0 & $9.940 \times 10^{-3}$ & $1.569 \times 10^{-3}$ & $2.348 \times 10^{-4}$ & 1 \\
5.0 & 0.1599 & $9.314 \times 10^{-2}$ & $5.088 \times 10^{-2}$ & 1 \\
\hline
\end{tabular}


$D \rightarrow D_{c}$ ) can be approached by decreasing the value of $r$ while increasing the size of the lattice, at finite lattice spacing. This provides a nonperturbative definition of the chiral limit for any $D$ of the general solution (3) with $D_{c}$ satisfying the necessary physical requirements [3].

It is evident that the range of proper values of $r$ also depends on the background gauge configuration. However, we suspect that when the background gauge configuration becomes very rough, there may not exist any values of $r$ such that the chiral anomaly function is in good agreement with the ChernPontryagin density. We intend to return to this question in a later publication.

In summary, we have clarified the role of $R$ in the general solution (3) of the Ginsparg-Wilson relation. It provides a topologically invariant transformation which transforms the chirally symmetric and nonlocal $D_{c}$ into a local $D$ which satisfies the GW relation, the exact chiral symmetry on the lattice. Having $R$ local in the position space is a necessary condition to ensure the absence of additive mass renormalization in the fermion propagator, as well as to produce a local $D$, which is vital for obtaining the correct chiral anomaly. Our numerical results strongly suggest that the optimal form of $R$ is $R(x, y)=r \delta_{x, y}$. The range of proper values of $r$ depends on the background gauge configuration as well as the size of the lattice, $L=N a$. In the limit $N \rightarrow \infty$, for smooth gauge backgrounds, the lower bound of proper values of $r$ goes to zero, thus the chiral limit $(r \rightarrow 0$ and $D \rightarrow D_{c}$ ) can be approached nonperturbatively at finite lattice spacing.

\section{Acknowledgements}

I would like to thank all participants of Chiral '99, workshop on chiral gauge theories (Taipei, Sep. 13-18, 1999), for their stimulating questions and interesting discussions. I am also indebted to Herbert Neuberger for his helpful comments on the first version of this paper. This work was supported by the National Science Council, ROC under the grant number NSC89-2112-M002-017.

\section{References}

[1] P. Ginsparg, K. Wilson, Phys. Rev. D 25 (1982) 2649.

[2] T.W. Chiu, S.V. Zenkin, Phys. Rev. D 59 (1999) 074501.

[3] T.W. Chiu, Phys. Lett. B 445 (1999) 371.

[4] T.W. Chiu, Phys. Lett. B 467 (1999) 112.

[5] H.B. Nielsen, N. Ninomiya, Nucl. Phys. B 185 (1981) 20 [E: B 195 (1982) 541]; B 193 (1981) 173.

[6] T.W. Chiu, Nucl. Phys. B (Proc. Suppl.) 73 (1999) 688, hep-lat/9809085.

[7] T.W. Chiu, Fermion determinant and chiral anomaly on a finite lattice hep-lat/9906007.

[8] H. Neuberger, Phys. Lett. B 417 (1998) 141; B 427 (1998) 353.

[9] T.W. Chiu, Phys. Rev. D 58 (1998) 074511. 